Article

\title{
Performance of Eudragit Coated Whispering Gallery Mode Resonator-Based Immunosensors
}

\author{
Ambra Giannetti ${ }^{1}$, Simone Berneschi ${ }^{1,2}$, Francesco Baldini ${ }^{1}$, Franco Cosi ${ }^{1}$, \\ Gualtiero Nunzi Conti ${ }^{1}$ and Silvia Soria ${ }^{1, *}$ \\ 1 Istituto di Fisica Applicata Nello Carrara (IFAC CNR), Via Madonna del Piano 10, \\ 50019 Sesto Fiorentino, Firenze, Italy; E-Mails: a.giannetti@ifac.cnr.it (A.G.); \\ s.berneschi@ifac.cnr.it (S.B.); f.baldini@ifac.cnr.it (F.B.); f.cosi@ifac.cnr.it (F.C.); \\ g.nunziconti@ifac.cnr.it (G.N.C.) \\ 2 Centro Studi e Ricerche e Museo Storico della Fisica Enrico Fermi, Piazza del Viminale 1, \\ 00184 Roma, Italy \\ * Author to whom correspondence should be addressed; E-Mail: s.soria@ifac.cnr.it; \\ Tel.: +39-055-522-6391; Fax: +39-055-522-6319.
}

Received: 26 September 2012; in revised form: 24 October 2012 / Accepted: 25 October 2012 / Published: 30 October 2012

\begin{abstract}
Whispering gallery mode resonators (WGMR) are an efficient tool for the realization of optical biosensors. A high $\mathrm{Q}$ factor preservation is a crucial requirement for good biosensor performances. In this work we present an Eudragit ${ }^{\circledR}$ L100 coated microspherical WGMR as an efficient immunosensor. The developed resonator was morphologically characterized using fluorescence microscopy. The functionalization process was tuned to preserve the high $\mathrm{Q}$ factor of the resonator. The protein binding assay was optically characterized in terms of specificity in buffer solution.
\end{abstract}

Keywords: whispering gallery mode resonators; immunosensors; Eudragit

\section{Introduction}

In optical bioassays, label-based assays exploit the interaction between the analyte under study and a capturing element, labelled with a fluorescent or chemiluminescent molecule. On the other hand, the use of a label very often implies multistep detection protocols (as in the ELISA tests or in sandwich assays) which can complicate the biochemical interaction and can cause sensor cross-sensitivities. On 
the contrary, in a label-free approach, which is based on the change of the refractive index in the medium surrounding the optical waveguide, does not suffer this inconvenience and offers the possibility to measure the interaction between the capturing element and the analyte directly and in real time, providing the possibility of also investigating dynamic interactions.

Detection of immunoagents and pathogens in a medical context requires the development of both highly sensitive and selective biosensors. This increasing demand for reliable detection of biomolecules has resulted in a large variety of optical label-free biosensors [1]. Among optical biosensors, evanescent wave based sensors are the most outstanding sensor platforms due to their capability of detecting changes induced by the binding of analytes within a submicron penetration depth (10 s to $100 \mathrm{~s}$ of $\mathrm{nm}$ ). Whispering Gallery Mode Resonators (WGMRs) offer a very promising alternative for the development of highly sensitive label-free biosensors [1-6], with the change in $Q$ or in resonant wavelength being used for measuring binding phenomena on the WGMR surface. It is apparent that high quality factor $Q$ and long recirculation of light in compact WGMRs are the most important features for sensing applications.

The permanence of high $Q$ values after the functionalization of the surface is an essential requirement in order to achieve highly sensitive biosensors. There is wide variety of surface chemistry already established for silica based WGMRs [7-9]. Recently, we investigated the effect of polymeric materials on microspherical WGMRs' $Q$ factors [10] following a procedure published in [11,12]. In this paper, we have effectively immobilized IgG and studied the resulting functionalized resonator morphologically by means of fluorescence microscopy. Afterwards, we have tested the performance of the WGMR immunosensors in a fluidic cell.

\section{Experimental Section}

\subsection{Materials}

Bovine serum albumin (BSA), ethanol (EtOH), phosphate-buffered saline (40 mM PBS, pH 7.4) and ethanolamine were purchased from Sigma-Aldrich (Milan, Italy). The methacrylic acid/methacrylate copolymer Eudragit ${ }^{\circledR} \mathrm{L} 100\left(\mathrm{n}_{\mathrm{D}}{ }^{20}=1.390-1.395\right)$ was purchased from Degussa, Röhm Pharma Polymers (Düsseldorf, Germany). The mouse IgG, goat anti-mouse-IgG and alexafluor488-labeled goat anti-mouse-IgG were purchased from Zymed Laboratories, Invitrogen Immunodetection (Milan, Italy). 1-Ethyl-3-[3-dimethylaminopropyl] carbodiimide hydrochloride (EDC) and $N$-hydroxysuccinimide (NHS) were purchased from Pierce (Rockford, IL, USA).

\subsection{Surface Functionalization and Bioassay Protocol}

Microspheres were treated by immersion in $10 \mathrm{mM}$ Eudragit ${ }^{\circledR} \mathrm{L} 100$ in ethanol for $1 \mathrm{~min}$ and then in exposed to air for about $15 \mathrm{~min}$, until complete solvent evaporation had occurred. The polymeric deposition provides carboxylic functional groups $(-\mathrm{COOH})$ on the surfaces, useful for biomolecule immobilization. Once the microspheres were functionalised, they were placed in a flow cell (see Section 2.3) and all the subsequent steps for the preparation of the biological sensing layer were performed: (i) activation of $-\mathrm{COOH}$ groups by means of EDC (2 mM) and NHS (5 mM) for $30 \mathrm{~min}$; (ii) immediate covalent immobilization of the antibodies on the microsphere surface, by pumping a 
solution of $100 \mathrm{mg} \cdot \mathrm{L}^{-1}$ antibody (Alexafluor488-labelled goat anti-mouse-IgG when the layer formation was investigated, mouse $\operatorname{IgG}$ when the bioassay was performed) in PBS for $1 \mathrm{~h}$; (iii) washing with PBS for 5 min to remove the unreacted antibody. After this step the microspheres with Alexafluor488-labelled goat anti-mouse-IgG were ready for measurements. The microspheres with mouse IgG were subject to further treatments; (iv) inactivation of carboxylic groups with $1 \mathrm{M}$ ethanolamine for $10 \mathrm{~min}$; (v) washing with PBS for $5 \mathrm{~min}$; (vi) surface passivation with 10\% BSA in PBS for $15 \mathrm{~min}$ in order to reduce/eliminate the non-specific adsorption on the glass surface; (vii) washing with PBS for $5 \mathrm{~min}$; (viii) injection for $30 \mathrm{~min}$ of Alexafluor488-labelled goat anti-mouse-IgG $50 \mathrm{mg} \cdot \mathrm{L}^{-1}$ in order to monitor optically the specific antigen/antibody interaction.

\subsection{Experimental Set-up}

Microspheres can easily be fabricated directly on the tip of a standard telecom fiber. For this purpose we used a fiber fusion splicer (FITEL S182K). A cleaved tip of the fiber is inserted in one arm of the splicer and a series of arcs are then produced. The tip partially melts and surface tension forces produce the spherical shape. The size of the spheres increases with the number of arc shots, up to a diameter saturation value of around $350 \mu \mathrm{m}$ [13]. The microspheres used in this work have an average diameter of $260 \mu \mathrm{m}$ and were stored under vacuum, in order to avoid contamination. The residual fiber stem is then mounted on a translation stage with piezoelectric actuators and a positioning resolution of $20 \mathrm{~nm}$.

The laser light is coupled to the WGM resonator by means of a tapered fiber of about $3 \mu \mathrm{m}$ diameter, produced in-house too. We used a microfluidic flow system that incorporates the WGMR and the tapered optical fiber. Our system consists of an open cell of $3 \mathrm{~mL}$ of volume with static flow. The tapered fiber was bound to the bottom of the cell by UV adhesive and placed parallel to the flow. The microsphere was then slowly lowered down to the contact point with the thinnest region of the taper and aligned at the centre of the fluidic cell. A peristaltic pump was used for filling the cell and injecting the antigen into the solution. A tunable external cavity laser (NetTest Tunics Plus), characterised by a working wavelength around $1.5 \mu \mathrm{m}$ and a linewidth of $300 \mathrm{kHz}$, is finely and slowly $(180 \mathrm{~Hz})$ modulated in wavelength around a WGM resonance of a few GHz. The light transmitted through the coupler-WGM resonator system was monitored with the pump off, at the output of the tape, using an amplified In GaAs photodiode detector connected to an oscilloscope, as shown in Figure 1.

The $Q$ values were obtained by measuring the resonance linewidth of the WGM modes around $1,550 \mathrm{~nm}$. The $Q$ factor of the WGMRs was monitored throughout the functionalization process to ensure that the chemical process and subsequent attachment of proteins preserved its optical performance [9].

\section{Results and Discussion}

\subsection{Optical Characterization}

The different steps of the protocol followed for the immobilization of the capturing element as well as for the bioassay implementation were morphologically characterized using a fluorescence microscope (Nikon Eclipse E600), thanks to the use of labeled antibodies, as described in Section 2.2. Table 1 lists the samples characterized by fluorescence microscopy and their relative $Q$ factors measured in buffer solution. 
Figure 1. A schematic diagram of the experimental arrangement.

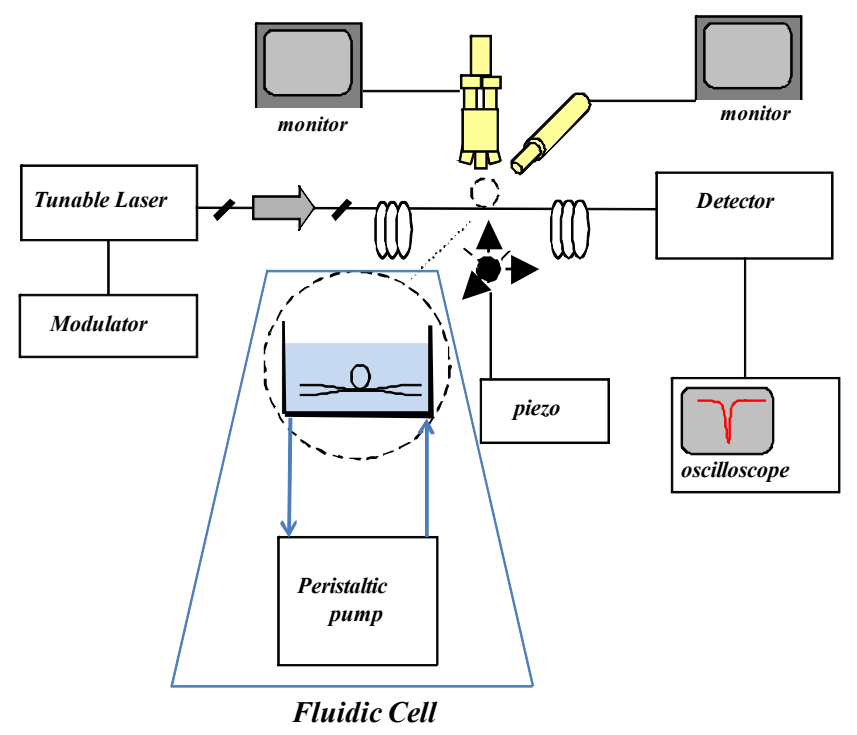

Table 1. Description of the samples used for optical characterization and their $Q$ factor.

\begin{tabular}{lll}
\hline Sample & Description & Q Factor \\
\hline NT & Non-treated sample in air & $2 \times 10^{8}$ \\
EU & Eudragit coated sample & $5.6 \times 10^{6}$ \\
IgG & Eudragit and Alexa488 mouse IgG sample & $3.6 \times 10^{5}$ \\
AntilgG & Eudragit, mouse IgG and Alexa488 goat anti-mouse IgG sample & $1.5 \times 10^{5}$ \\
\hline
\end{tabular}

In order to evaluate the distribution of IgG on microspheres, the Alexa488 goat anti-mouse-IgG was immobilized on Eudragit coated microspheres using the protocol described above. The microsphere was immobilized on a microscope slide with a double-sided tape in order to reduce the lateral shift. In order to confirm the bio-recognition event, observations of anti-mouse-IgG modified microspheres incubated with labeled protein solutions were carried out.

The anti-mouse-IgG distribution on the microsphere was found to be quite uniform, in particular in the equatorial region, as shown in Figure 2. From optical images and the measured $Q$ factor is apparent that Eudragit functionalization induces a uniform modification of the surface of the microspheres. The $Q$-factor preservation allowed efficient protein target recognition, as reported in the optical biosensor detection section [10]. The measured $Q$ factor after $\operatorname{IgG} /$ anti-IgG binding is $1.5 \times 10^{5}$ at $1,550 \mathrm{~nm}$ in PBS. Figure 3 shows the measured $Q$ factor for an NT sample, an IgG one and the last one after IgG/anti-IgG binding.

Figure 2. Fluorescent image of the sample anti-mouse-IgG.

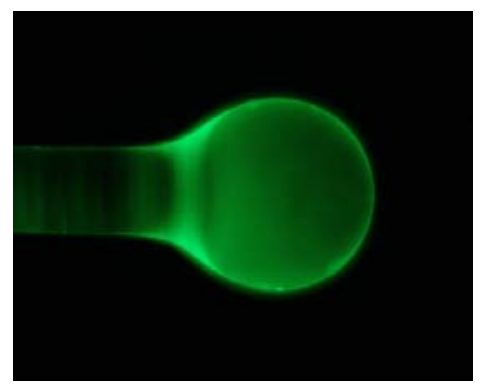


Figure 3. Measured $Q$ factor of the NT sample (black line), of a covalent binding of IgG (blue line) and an IgG/anti-IgG interaction (red line).

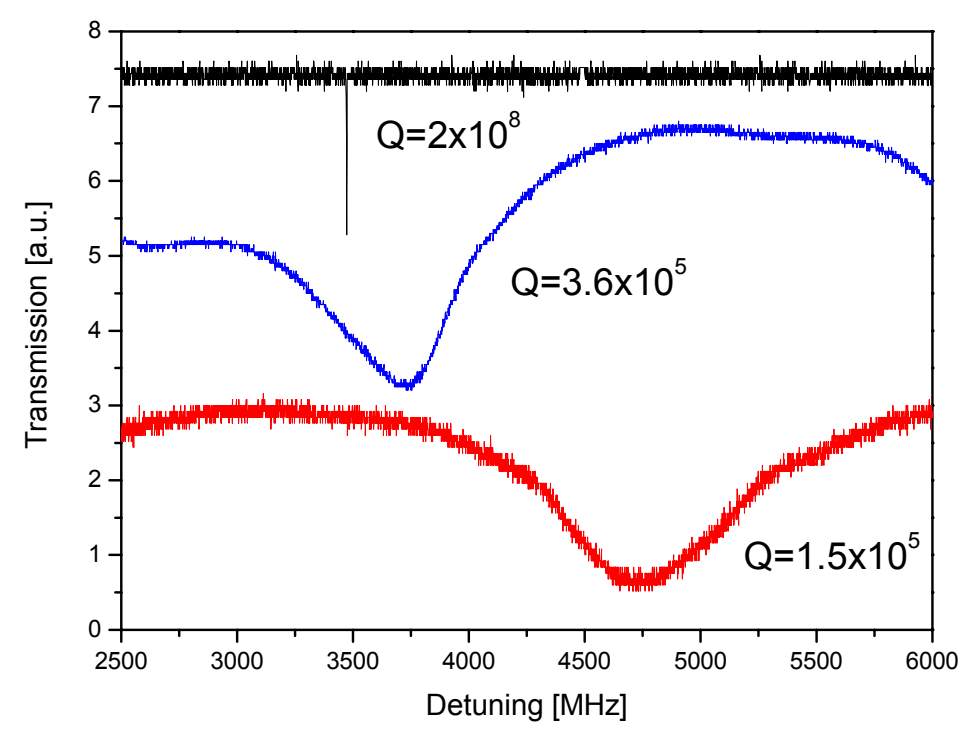

\subsection{Binding Assay}

In order to study the feasibility of using the Eudragit coated microsphere for immunosensing applications, we carried out a preliminary experiment in which we injected $10 \mu \mathrm{L}$ of $50 \mathrm{mg} \cdot \mathrm{L}^{-1}$ Alexafluor488-labelled goat anti-mouse-IgG so as to monitor the specific antigen/antibody interaction. Figure 4 shows the sensorgram, illustrating that the response of the WGMR immunosensor after goat anti-mouse-IgG injection is specific.

Figure 4. Sensorgram of anti-IgG binding to a WGMR immobilized with IgG in PBS, following the addition of anti-IgG.

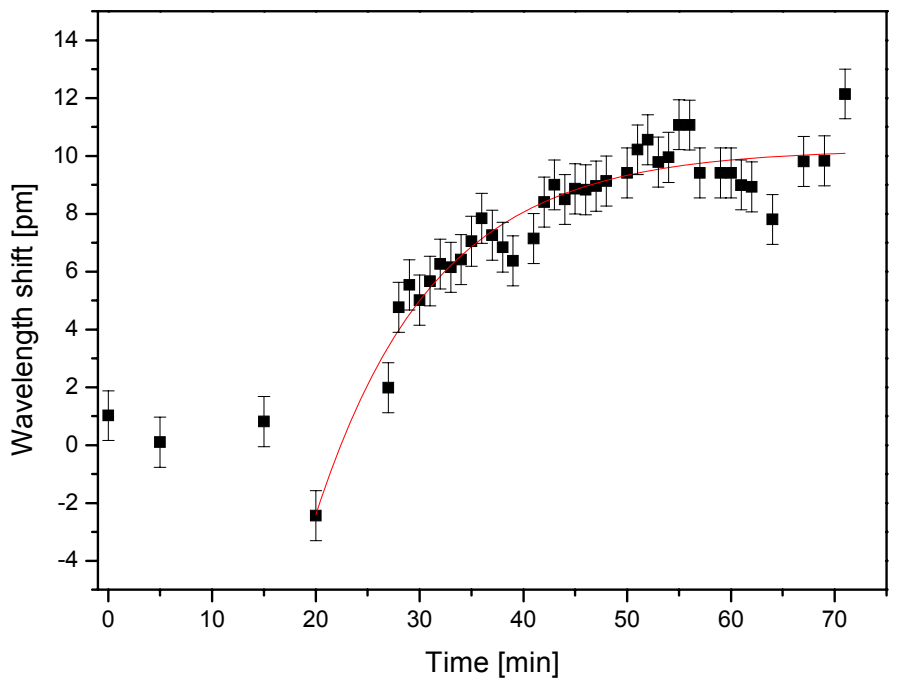

The wavelength shift is first negative due to thermal effects $[13,14]$, but rapidly reverses as the resonant wavelength begins to increase and saturates with time. The sensor to sensor performance was highly reproducible when using freshly prepared samples. The experiments were repeated several times with similar results. For the IgG/anti-IgG binding experiment, we have measured a wavelength 
shift of about $10.2 \pm 0.3 \mathrm{pm}$. Wavelength shifts are related to surface density of bound molecules by the following equation $[15,16]$ :

$$
\delta \lambda / \lambda=\alpha_{\mathrm{ex}} \sigma /\left[\varepsilon_{0}\left(\mathrm{n}_{\mathrm{s}}^{2}-\mathrm{n}_{\mathrm{m}}^{2}\right) \mathrm{a}\right]
$$

where $n_{s}$ and $n_{m}$ are the refractive indices of the microsphere and the medium, respectively, a is the radius of the microsphere, $\alpha_{e x}$ is the excess of polarizability, and $\sigma$ is the surface density of molecules forming a layer.

The excess of polarizability for IgG can be calculated from [16]:

$$
\alpha_{\mathrm{ex}}=2 \times \varepsilon_{0} \times \mathrm{n}_{\mathrm{m}} \times(\mathrm{dn} / \mathrm{dc}) \times \mathrm{M}_{\mathrm{w}} / \mathrm{n}_{\mathrm{A}}
$$

where $\mathrm{dn} / \mathrm{dc}$ is the differential refractive index of a protein solution in water, $\sim 0.184 \mathrm{~cm}^{3} / \mathrm{g}$ [17], $\mathrm{M}_{\mathrm{w}}=150 \mathrm{kDa}$ is the molecular weight of $\mathrm{IgG}$ and $\mathrm{n}_{\mathrm{A}}$ is the Avogadro's number. With $\alpha_{\mathrm{ex}}=4 \pi \varepsilon_{0} \times 9.85 \times 10^{-21} \mathrm{~cm}^{3}, \mathrm{a}=130 \mu \mathrm{m}$ (the average microsphere radius used in these experiments) and $\delta \lambda=10.2 \mathrm{pm}$, the anti-IgG surface density is estimated to be $2.25 \times 10^{11} \mathrm{~cm}^{-2}$, which is close to a compact layer. Although IgG is a globular protein, it is difficult to predict the exact conformation and hence its size when adsorbed to a surface, although we assume that it binds with its largest planar projection in order to reduce the surface energy. Crystallographic and TEM data give an upper limit of approximately 16 to $19 \mathrm{~nm}[18,19]$. Assuming $\mathrm{IgG}$ as a roughly spherical molecule of about $17.5 \mathrm{~nm}$ of diameter [20], its projected area is then about $240 \mathrm{~nm}^{2}$. The maximum fractional area coverage is estimated to be 0.55 for random packing of spheres [21] which means that $\sigma_{\max }=2 \times 10^{11} \mathrm{~cm}^{-2}$ for IgG. Thus, our IgG layer is about the theoretical level.

Binding of kinetics could be fit to a simple model. The signal of the WGMR biosensor, neglecting the dissociation rate constant, can be modelled by a differential equation, whose solution is a simple exponential [22]:

$$
\delta \lambda=\delta \lambda_{\max }\left[1-\exp \left(-k_{\mathrm{a}} \mathrm{Ct}\right)\right]
$$

where $\mathrm{C}$ is the concentration and $k_{a}$ is the association constant. In this simple model, we have neglected transport phenomena. From the experimental data, assuming that binding is saturated with the used concentration, we obtained that the association constant $k_{a}$ is about $1.9 \times 10^{5} \mathrm{M}^{-1} \mathrm{~s}^{-1}$ for $\operatorname{IgG}$, which are in good accordance with published results for IgG/anti-IgG binding, based on fiber optics biosensors [23] but 10-fold lower than those obtained using conventional methods [24].

\section{Conclusions}

In conclusion, we have demonstrated a WGMR based immunosensor. We have studied and developed the most suitable chemical protocol in order to obtain a homogeneous thin polymeric layer. Subsequent chemical activation and covalent binding of globular proteins like IgG preserve a value of $Q$ high enough for immunosensing applications. We have performed a series of morphological studies in order to assess the quality of the surfaces and determine whether the covalent binding is achieved. It was also shown that WGMR sensors can be used for determining binding kinetics. 


\section{Acknowledgments}

S. Berneschi fully acknowledges funding from Centro Studi e Ricerche "E. Fermi". Financial support by PAT-NAoMI Project is gratefully acknowledged. Support by NoE "Photonics 4 Life" is also acknowledged.

\section{References}

1. Fan, X.; White, I.M.; Shopova, S.I.; Zhu, H.; Suter, J.D.; Sun, Y. Sensitive optical biosensors for unlabeled targets: A review. Anal. Chim. Acta 2008, 620, 8-26.

2. Luchansky, M.S.; Bailey, R.C. High Q optical sensors for chemical and biological analysis. Anal. Chem. 2012, 84, 793-821.

3. Soria, S.; Berneschi, S.; Brenci, M.; Cosi, F.; Nunzi Conti, G.; Pelli, S.; Righini, G.C. Optical Microspherical Resonators for Biomedical Sensing. Sensors 2011, 11, 785-805.

4. Ilchenko, V.S.; Matsko, A.B. Optical Resonators with Whispering-Gallery Modes-Part II: Applications. IEEE J. Sel. Top. Quantum Electron. 2006, 12, 15-32.

5. Vollmer, F.; Arnold, S. Whispering-gallery-mode biosensing: Label-free detection down to single molecules. Nat. Methods 2008, 5, 591-596.

6. Chiasera, A.; Dumeige, Y.; Féron, P.; Ferrari, M.; Jestin, Y.; Nunzi Conti, G.; Pelli, S.; Soria, S.; Righini, G.C. Spherical whispering-gallery-mode microresonators. Laser Photonics Rev. 2010, 4 , 457-483.

7. Vollmer, F.; Arnold, S.; Braun, D.; Teraoka, I.; Libchaber, A. Multiplexed DNA Quantification by Spectroscopic Shift of Two Microsphere Cavities. Biophys. J. 2003, 85, 1974-1979.

8. Zhu, H.; Suter, J.D.; White, I.M.; Fan, X. Aptamer Based Microsphere Biosensor for Thrombin Detection. Sensors 2006, 6, 785-795.

9. Pasquardini, L.; Berneschi, S.; Barucci, A.; Cosi, F.; Dallapiccola, R.; Insinna, M.; Lunelli, L.; Nunzi Conti, G.; Pederzolli, C.; Salvadori, S.; et al. Whispering gallery mode aptasensors for detection of blood proteins. J. Biophotonics 2012, doi:10.1002/jbio.201200013.

10. Soria, S.; Baldini, F.; Berneschi, S.; Cosi, F.; Giannetti, A.; Nunzi Conti, G.; Pelli, S.; Righini, G.C.; Tiribilli, B. High-Q polymer-coated microspheres for immunosensing applications. Opt. Express 2009, 17, 14694-14699.

11. Baldini, F.; Carloni, A.; Giannetti, A.; Porro, G.; Trono, C. A new optical platform for biosensing based on fluorescence anisotropy. Anal. Bioanal. Chem. 2008, 391, 1837-1844.

12. Baldini, F.; Carloni, A.; Giannetti, A.; Porro, G.; Trono, C. An optical PMMA biochip based on fluorescence anisotropy: Application to C-reactive protein assay. Sens. Actuators B 2009, 139, 64-68.

13. Brenci, M.; Calzolai, R.; Cosi, F.; Nunzi Conti, G.; Pelli, S.; Righini, G.C. Microspherical resonators for biophotonic sensors. Proc. SPIE 2006, 6158, 61580S.1-61580S.9.

14. Ma, Q.; Rossman, T.; Guo, Z. Whispering-gallery mode silica microsensors for cryogenic to room temperature measurement. Meas. Sci. Technol. 2010, 21, 025310.

15. Arnold, S.; Khoshsima, M.; Teraoka, I.; Holler, S.; Vollmer, F. Shift of Whispering Gallery Modes in Microspheres by Protein Adsorption. Opt. Lett. 2003, 28, 272-274. 
16. Vollmer, F.; Braun, D.; Libchaber, A.; Khoshsima, M.; Teraoka, I.; Arnold, S. Protein Detection by Optical Shift of a Resonant Microcavity. Appl. Phys. Lett. 2002, 80, 4057-4059.

17. Beier, H.T.; Coté, G.L.; Meissner, K.E. Whispering Gallery Mode Biosensors Consisting of Quantum Dot-Embedded Microspheres. Ann. Biomed. Eng. 2009, 37, 1974-1983.

18. Brady, R.L.; Hubbard, R.E.; King, D.J.; Low, D.C.; Roberts, S.M.; Todd, R.J. Crystallization and preliminary X-ray diffraction study of a chimaeric Fab' fragment of antibody binding tumour cells. J. Mol. Biol. 1991, 219, 603-604.

19. Wrigley, N.G.; Brown, E.B.; Skehel, J.J. Electron microscopic evidence for the axial rotation and inter-domain flexibility of the fab regions of immunoglobulin G. J. Mol. Biol. 1983, 169, 771-774.

20. Roberts, C.J.; Davies, M.C.; Tendler, S.J.B.; Williams, P.M.; Davies, J.; Dawkes, A.C.; Yearwood, G.D.L.; Edwards, J.C. The discrimination of IgM and IgG type antibodies and Fab' and $F^{\prime}(a b) 2$ antibody fragments on an industrial substrate using scanning force microscopy. Ultramicroscopy 1996, 62, 145-149.

21. Torquato, S. Mean Nearest-Neighbor Distance in Random Packings of Hard D-Dimensional Spheres. Phys. Rev. Lett. 1995, 74, 2156-2159.

22. Lin, Y.; Ilchenko, V.S.; Nadeau, J.; Maleki, L. Biochemical detection with optical whisperinggallery resonators. Proc. SPIE 2007, 6452, doi:10.1117/12.716591.

23. Chou, C.; Wu, H.-T.; Yu, C.-J.; Chang, Y.-F.; Hsieh, B.-Y. Fiber-optic biosensor for antigen/antibody kinetic assays. SPIE Newsroom 2007, doi:10.1117/2.1200707.0768.

24. Ivnitski, D.; Wolf, T.; Solomon, B.; Fleminger, G.; Rishpon, J. An amperometric biosensor for real-time analysis of molecular recognition. Bioelectrochem. Bioenerg. 1998, 45, 27-35.

(C) 2012 by the authors; licensee MDPI, Basel, Switzerland. This article is an open access article distributed under the terms and conditions of the Creative Commons Attribution license (http://creativecommons.org/licenses/by/3.0/). 